\title{
BMJ Open Football's InfluencE on Lifelong health and Dementia risk (FIELD): protocol for a retrospective cohort study of former professional footballers
}

\author{
Emma R Russell, ${ }^{1}$ Katy Stewart, ${ }^{2,3}$ Daniel F Mackay, ${ }^{4}$ John MacLean, ${ }^{2,3}$ Jill P Pell, ${ }^{4}$ \\ William Stewart ${ }^{1,5}$
}

To cite: Russell ER, Stewart K, Mackay DF, et al. Football's InfluencE on Lifelong health and Dementia risk (FIELD): protocol for a retrospective cohort study of former professional footballers. BMJ Open 2019;9:e028654. doi:10.1136/ bmjopen-2018-028654

- Prepublication history for this paper is available online. To view these files, please visit the journal online (http://dx.doi. org/10.1136/bmjopen-2018028654).

Received 18 December 2018 Revised 26 March 2019 Accepted 4 April 2019

\section{Check for updates}

(C) Author(s) (or their employer(s)) 2019. Re-use permitted under CC BY-NC. No commercial re-use. See rights and permissions. Published by BMJ.

${ }^{1}$ Glasgow Brain Injury Research Group, Institute of Neuroscience and Psychology, University of

Glasgow, Glasgow, UK

${ }^{2}$ Sport and Exercise Medicine, Institute of Cardiovascular and Medical Sciences, University of Glasgow, Glasgow, UK

${ }^{3}$ Hampden Sports Clinic, Hampden Stadium, Glasgow, UK ${ }^{4}$ Public Health, Institute of Health and Wellbeing, University of Glasgow, Glasgow, UK ${ }^{5}$ Department of Neuropathology, Queen Elizabeth University Hospital, Glasgow, UK

Correspondence to Dr William Stewart; william.stewart@glasgow.ac.uk

\section{ABSTRACT}

Introduction In the past decade, evidence has emerged suggesting a potential link between contact sport participation and increased risk of late neurodegenerative disease, in particular chronic traumatic encephalopathy. While there remains a lack of clear evidence to test the hypothesis that contact sport participation is linked to an increased incidence of dementia, there is growing public concern regarding the risk. There is, therefore, a pressing need for research to gain greater understanding of the potential risks involved in contact sports participation, and to contextualise these within holistic health benefits of sport.

Methods and analysis Football's InfluencE on Lifelong health and Dementia risk is designed as a retrospective cohort study, with the aim to analyse data from former professional footballers (FPF) in order to assess the incidence of neurodegenerative disease in this population. Comprehensive electronic medical and death records will be analysed and compared with those of a demographically matched population control cohort. As well as neurodegenerative disease incidence, all-cause, and disease-specific mortality, will be analysed in order to assess lifelong health. Cox proportional hazards models will be run to compare the data collected from FPFs to matched population controls.

Ethics and dissemination Approvals for study have been obtained from the University of Glasgow College of Medical, Veterinary and Life Sciences Research Ethics Committee (Project Number 200160147) and from National Health Service Scotland's Public Benefits and Privacy Panel (Application 1718-0120).

\section{INTRODUCTION}

Approximately 850000 people in the UK are living with dementia, with an annual cost to society of £26 billion. ${ }^{1}$ Identifying possible risk factors for neurodegenerative disease and acting to reduce these is a priority. In the past decade, concerns around perceived increased dementia risk through participation in contact sports have led to numerous news headlines, ${ }^{2}$ a Hollywood movie ${ }^{3}$ and demands from media and governments for
Strengths and limitations of this study

- Utilising a comprehensive dataset of former professional footballers (FPF) and matched population controls (MPC) minimises risks of bias.

- Accessing complete electronic health records, Football's InfluencE on Lifelong health and Dementia risk has the opportunity to look at holistic lifelong health outcomes from professional football.

- Establishing a robust dataset on the primary aims will inform study design and target sample size for future research enquiries in this field.

- Errors in health records' coding are widely recognised, but are assumed to be consistent between FPF and MPC.

- As a retrospective investigation of existing electronic health records data on specific risk factors for neurodegeneration of interest, in particular exposure to traumatic brain injury, will be limited. These will require to be addressed in follow-on studies using different methodologies.

research into the issues raised. In part, this has been driven by growing recognition of a specific form of neurodegenerative pathology thought to be a consequence of exposure to traumatic brain injury (TBI), chronic traumatic encephalopathy (CTE), ${ }^{4}$ in autopsy studies of athletes from a wide range of contact sports, including American football, association football (soccer; hereafter 'football') and rugby. ${ }^{5-7}$ Nevertheless, despite this attention, there remain limited data to inform on the lifelong neurological consequences of participation in contact sport, including dementia risk.

Regarding football, CTE pathology (then referred to as dementia pugilistica) was first reported in a former amateur footballer at the end of the last century. ${ }^{8}$ Since then, seven further published case descriptions of CTE neuropathology in former footballers have been reported. ${ }^{5}$ However, issues of case 
selection bias and reporting limitations in these autopsy studies mean the few cases reported so far provide no insight into incidence of CTE in former footballers, nor do they inform on risk of neurodegenerative disease in this population.

Beyond CTE, data on the long-term neurological consequences of participation in sport are limited and conflicting. Thus, regarding American Football, while one study reported a threefold increase in neurodegenerative mortality in a cohort of former National Football League players, ${ }^{9}$ studies in former high school American footballers failed to identify any increased dementia risk. ${ }^{10}$ Regarding late neurocognitive impact of participation in contact sport, mild, subclinical deficits in limited neuropsychological assessments have been documented in former elite rugby union players when compared with matched controls; ${ }^{11}$ observations since replicated in other studies. ${ }^{12}$ In American football, a study of retired players concluded that those who had suffered multiple concussions were more likely to be diagnosed with some degree of cognitive impairment. ${ }^{13}$

In association football, incidence of amyotrophic lateral sclerosis (ALS) has been documented as higher in former Italian professional footballers than expected from population statistics. ${ }^{14}$ However, methodological limitations, including just five ALS cases in their study population of over 7000 , and failure to use appropriately matched population data, mean these results must be interpreted with caution. Elsewhere, a separate study reported $11 \%$ of former footballers screening positive for cognitive impairment in a self-assessment survey, which is in line with population expectations. ${ }^{15}$ Again, however, interpretation of this study is hampered by significant methodological limitations, including modest recruitment numbers, selection bias in self-reporting of symptoms and failure to identify an appropriately matched community control population. Therefore, given the lack of robust, adequately powered studies with appropriate population controls no meaningful conclusion on late neurodegenerative disease risk in former athletes, including footballers can be drawn.

While the late neurocognitive consequences of participation in sport remain uncertain, the health benefits of physical activity are long established. Specifically, regular physical activity is recognised as a benefit in both primary and secondary prevention of a range of chronic diseases, ${ }^{16}$ including dementia, ${ }^{17}$ and in reducing risk of premature death ${ }^{16}$ findings promoted by Public Health England ${ }^{18}$ and the Center for Disease Control. ${ }^{19}$ Indeed, while studies in late neurocognitive outcomes in sport provide limited insight regarding their primary aims, by contrast they provide consistent support for lifelong health benefits of sports participation. Thus, in reporting increased neurodegenerative mortality in former NFL footballers the authors also noted reduced all-cause mortality, ${ }^{9}$ while others report reduced cardiovascular disease in former rugby internationalists compared with their matched controls. ${ }^{11}$
Given growing public anxieties over perceived increased risk of dementia in former professional athletes, in particular former footballers, establishing robust data to inform on late neurodegenerative outcomes of participation in contact sports is a priority to inform public health policy and sports' governing organisations. Importantly, however, insight into risk of neurodegenerative disease must be read in context of wider lifelong health outcomes to permit adequately informed discussions on the holistic public health implications of sport. Using established protocols that have proven highly successful in addressing similar research aims in other populations of interest. ${ }^{20-22}$ Football's InfluencE on Lifelong health and Dementia risk (FIELD) is designed as a retrospective cohort study looking at a wide range of physical and mental health outcomes, including neurodegenerative disease, in former professional footballers (FPF)compared with an age, sex and deprivation matched general population comparison group. The specific aims of the FIELD study are:

- To compare the incidence of neurodegenerative disease, including dementia, in FPF to a matched general population comparison group.

- To compare the incidence of chronic physical and mental health conditions in FPF to a matched general population comparison group.

- To compare all-cause and disease-specific mortality in FPF to a matched general population comparison group.

- To register former footballers for autopsy brain donation for diagnostic assessment at the time of death to inform on neurodegenerative pathologies in this population.

\section{METHODS AND ANALYSIS}

\section{Cohort identification}

FPF will be identified from independent databases of all Scottish professional footballers held as the Record of Pre-war Scottish League Players (v2) and the Record of Post-war Scottish League Players (v6). These databases comprise comprehensive datasets of information on every player for all Scottish League clubs and have been compiled from the archives of the Scottish Football Museum and the individual league clubs. Included in these datasets are basic player demographics (full name and date of birth), together with extensive career information (including, where available, date of first signing and retirement, number of match appearances and player position). From these databases, duplicate entries (eg, player recorded by more than one club over career) will be merged to a single player record and a cohort of FPF will be identified as: full name and date of birth available; and age 40 years or over at 31 December 2016. Exclusion criteria will be: incomplete demographic information and age under 40 years at 31 December 2016. The selection of an age cut-off of 40 years reflects the suggestion that there might be an earlier age at onset for neurodegenerative disease in former athletes, ${ }^{4}$ while also acknowledging that prevalence 
of such disease is extremely low below this age. ${ }^{23}$ Alongside basic demographic information, data to support potential secondary analyses will be extracted, including years of playing professional football and player position.

MPC will be selected matched to age, sex and deprivation for the FPF cohort on a 3:1 basis (ie, for every FPF, three MPC will be identified). The Information Services Division of NHS Scotland supports the electronic Data Research and Innovation Service (eDRIS) ${ }^{24}$ This database also records last known postcode of residence for all individuals in Scotland, which is used to provide information on area socioeconomic deprivation based on the Scottish Index of Multiple Deprivation (SIMD) ${ }^{24}$ The SIMD is derived from information on income, employment, health, education (including skills and training), housing and crime for each geographic area (by postcode), with the data commonly accessed in quintiles of socioeconomic status, ranging from 1 (most deprived) to 5 (least deprived).

\section{Health records interrogation}

In addition to information on socioeconomic deprivation as SIMD, eDRIS provides research support and access to comprehensive inpatient and outpatient electronic medical and death certification records prospectively from 1981 to present day for all Scottish residents. Using available demographic information (full name and date of birth) probability matching will be used to match each FPF to their unique Community Health Index number, which will then be used to provide individual level data for FPF and their MPC on: outpatient attendances (SMR00), admissions to general acute (SMR01) and psychiatric (SMR04) hospitals, cancer registrations (SMR06), and death certification. These records include diagnostic codes (International Classification of Diseases 9/10) identifying the condition resulting in hospitalisation or death. The FIELD study launches on 1 March 2018 and is scheduled to run for 36 months, to 31 January 2021.

\section{Autopsy registration}

Using existing, approved protocols FPF will be invited to register for research brain banking in the event of death via open calls for registration and participant information disseminated in partnership with supporting sports associations and charities (eg, The Jeff Astle Foundation). In the event of death, specimens will be retrieved using existing national neuropathology networks and protocols and directed to the unique, internationally recognised Glasgow TBI Archive for diagnostic evaluation and to support wider research access under the NHS Greater Glasgow and Clyde Bio-repository governance framework.

\section{Statistical analysis}

Separate Cox proportional hazards models will be run for each of the health outcomes comparing FPF and MPC. Analyses will run univariately, and will also be repeated after adjustment for potential confounders including player position and length of career. We will stratify by date of birth in order to look at birth cohort effects. We will also test for statistical interactions and undertake subgroup analyses where these are found. Predicting a population prevalence of neurodegenerative disease in the age range under study of 2.5 per 100 population $^{123}$ and an average disease duration of 15 years, then to detect a $50 \%$ increase in disease incidence will require a sample size of 5653 for $80 \%$ power (alpha $=0.05$ ), or 7613 for $90 \%$ power (Stata V.14, 2015; StataCorp).

\section{Patient and public involvement}

There was no direct patient or public involvement in the development of the FIELD study protocol.

\section{Limitations}

The study has some limitations. First, we have limited access to information on confounders beyond age, sex and deprivation status. Confounders such as family history, and genetic predisposition to certain diseases cannot be taken into account. Second, diagnostic coding is a limitation. We are restricted by how much information is supplied on death certificates and by medical records, the listed cause of death may not take into account contributing factors for death. Finally, the NHS was not founded until 1948, and we therefore may have less complete medical records or no medical records at all for some players born prior to this time period.

\section{Ethics and dissemination}

All health data returned to researchers will be anonymised with no return to original identifying information. As a secondary data analysis, individual consent from FPF and MPC is not required.

Based on previous, highly successful enquiries from our group using this same methodology, ${ }^{20-22}$ we anticipate this study will generate multiple informative manuscripts for publication in high-impact journals. As a priority, we intend outputs reporting: incidence of neurodegenerative disease in FPF in context of wider lifelong health outcomes; cause of death statistics in FPF and influence of player position and playing era on incidence of neurodegenerative disease in FPF. Further, we anticipate data will be discussed in preliminary formats at appropriate scientific conferences.

Contributors ERR produced the first draft of the manuscript and is responsible for data collection in FIELD; KS, DFM, JM and JPP are co-investigators on FIELD, Codesigned the study protocol and edited the manuscript; WS is principal investigator on FIELD, co-designed the study, produced the study proposal and protocols and edited the manuscript.

Funding This study is supported by funding from The Football Association and Professional Footballers' Association and an NHS Research Scotland Career Researcher Fellowship (W Stewart).

Competing interests None declared.

Patient consent for publication Not required.

Ethics approval Approval for this study has been sought and provided by the University of Glasgow College of Medical, Veterinary and Life Sciences Ethics Committee (Project Number 200160147). The FIELD study has also undergone proportionate governance review and been approved by NHS Scotland's Public Benefit and Privacy Panel for Health and Social Care (reference 1718 - 0120).

Provenance and peer review Not commissioned; externally peer reviewed. 
Open access This is an open access article distributed in accordance with the Creative Commons Attribution Non Commercial (CC BY-NC 4.0) license, which permits others to distribute, remix, adapt, build upon this work non-commercially, and license their derivative works on different terms, provided the original work is properly cited, appropriate credit is given, any changes made indicated, and the use is non-commercial. See: http://creativecommons.org/licenses/by-nc/4.0/.

\section{REFERENCES}

1. M K, Wittenberg R, Comas-Herrera A, et al. Dementia UK: Update. Igarss 2014. 2014.

2. Rutherford A, Stewart W, Bruno D. Heading for trouble: is dementia a game changer for football? Br J Sports Med 2019;53.

3. Smith DH, Stewart W. Tackling concussion, beyond Hollywood. Lancet Neurol 2016;15:662-3.

4. Smith DH, Johnson VE, Stewart W. Chronic neuropathologies of single and repetitive TBI: substrates of dementia? Nat Rev Neurol 2013;9:211-21.

5. Ling H, Morris HR, Neal JW, et al. Mixed pathologies including chronic traumatic encephalopathy account for dementia in retired association football (soccer) players. Acta Neuropathol 2017;133:337-52.

6. Stewart W, McNamara PH, Lawlor B, et al. Chronic traumatic encephalopathy: a potential late and under recognized consequence of rugby union? QJM 2016;109:11-15.

7. Omalu BI, DeKosky ST, Minster RL, et al. Chronic traumatic encephalopathy in a National Football League player. Neurosurgery 2005:57:128-34.

8. Geddes JF, Vowles GH, Nicoll JA, et al. Neuronal cytoskeletal changes are an early consequence of repetitive head injury. Acta Neuropathol 1999;98:171-8.

9. Lehman EJ, Hein MJ, Baron SL, et al. Neurodegenerative causes of death among retired National Football League players. Neurology 2012;79:1970-4.

10. Janssen PH, Mandrekar J, Mielke MM, et al. High school football and late-life risk of neurodegenerative syndromes, 1956-1970. Mayo Clin Proc 2017;92:66-71.
11. McMillan TM, McSkimming P, Wainman-Lefley J, et al. Long-term health outcomes after exposure to repeated concussion in elite level: rugby union players. J Neurol Neurosurg Psychiatry 2017;88.

12. Decq P, Gault N, Blandeau M, et al. Long-term consequences of recurrent sports concussion. Acta Neurochir 2016;158:289-300.

13. Guskiewicz KM, Marshall SW, Bailes J, et al. Association between recurrent concussion and late-life cognitive impairment in retired professional football players. Neurosurgery 2005;57:719-26.

14. Chiò A, Benzi G, Dossena M, et al. Severely increased risk of amyotrophic lateral sclerosis among Italian professional football players. Brain 2005;128:472-6.

15. Vann Jones SA, Breakey RW, Evans PJ. Heading in football, longterm cognitive decline and dementia: evidence from screening retired professional footballers. Br J Sports Med 2014;48:159-61.

16. Warburton DE, Nicol CW, Bredin SS. Health benefits of physical activity: the evidence. CMAJ 2006;174:801-9.

17. Rovio S, Kåreholt I, Helkala EL, et al. Leisure-time physical activity at midlife and the risk of dementia and Alzheimer's disease. Lancet Neurol 2005;4:705-11.

18. PHE. Public Health England. 2016 http://bit.ly/2a4FStR.

19. CDC. Centers for Disease Control and Prevention - Physical Activity. https://www.cdc.gov/physicalactivity/index.html.

20. Bergman BP, Mackay DF, Pell JP. Acute myocardial infarction in Scottish military veterans: a retrospective cohort study of 57,000 veterans and 173,000 matched nonveterans. Am J Epidemiol 2014;179:1434-41.

21. Bergman BP, Mackay DF, Pell JP. Motor neurone disease and military service: evidence from the Scottish Veterans Health Study. Occup Environ Med 2015;72:877-9.

22. Bergman BP, Mackay DF, Pell JP. Lymphohaematopoietic malignancies in Scottish military veterans: Retrospective cohort study of 57,000 veterans and 173,000 non-veterans. Cancer Epidemiol 2017;47:100-5.

23. Harvey RJ, Skelton-Robinson M, Rossor MN. The prevalence and causes of dementia in people under the age of 65 years. $J$ Neurol Neurosurg Psychiatry 2003;74:1206-9.

24. SIMD. The Scottish Index of Multiple Deprivation. http://www.gov. scot/Topics/Statistics/SIMD. 